\title{
Positive Predictive Value of the ICD-I0 Diagnosis Code for Long-COVID
}

\author{
Lærke Storgaard Duerlund (D)', Shakil Shakar², Henrik Nielsen (1D)', Jacob Bodilsen (D)' \\ 'Department of Infectious Diseases, Aalborg University Hospital, Aalborg, Denmark; ${ }^{2}$ The Department of Medicine, Regionshospitalet Nordjylland \\ Hjørring, Hjørring, Denmark \\ Correspondence: Jacob Bodilsen, Department of Infectious Diseases, Aalborg University Hospital, Mølleparkvej, 4, Aalborg, 9000, Denmark, \\ Tel +45 97660566, Email Jacob.bodilsen@rn.dk
}

Purpose: To examine the positive predictive value (PPV) of International Classification version 10 (ICD-10) diagnosis codes for long coronavirus disease 2019 (long-COVID) in a Danish Health registry.

Patients and Methods: This was a medical record review of all patients with a diagnosis code of long-COVID (DB948A) at all hospitals in the North Denmark Region from February 27, 2020 through June 30, 2021. Confirmed long-COVID was categorized as fulfillment of all three criteria: (1) a positive polymerase chain reaction (PCR) test for SARS-CoV-2 on a respiratory sample or a positive serum antibody test, (2) symptoms suggestive of long-COVID with no other diagnosis considered more likely, and (3) symptom duration $>6$ weeks.

Results: A total of 306 patients were assigned a hospital diagnosis code for long-COVID corresponding to $1.4 \%$ of all SARS-CoV-2 positive individuals during the study period $(n=21,727)$. Next, 40 patients were excluded due to incomplete diagnostic evaluation at time of record review leaving 266 patients for analysis. The patients had a median age of 51 years (interquartile range 43-60) and 175/ 266 (66\%) were females. Long-COVID was confirmed in 249/266 yielding an overall PPV of 94\% (95\%CI: 90-96) and did not differ substantially according to most age groups, sex, previous hospitalization for COVID-19, or by using 12 weeks of symptom duration as cut-off. The PPV was low for children and adolescents $(n=5)$, the very elderly $(n=9)$, and those included by secondary long-COVID diagnoses $(\mathrm{n}=10)$.

Conclusion: The overall PPV of diagnosis codes for long-COVID in the North Denmark Region was high and was likely suitable for future registry-based studies of long-COVID. Caution is advised at the extremes of age and secondary diagnosis codes.

Keywords: post-COVID, coronavirus disease 2019, diagnosis codes, validation, epidemiology

\section{Introduction}

Currently, more than 268 million people have been infected with severe acute respiratory syndrome coronavirus 2 (SARS-CoV-2) of which more than 5.3 million had a fatal outcome. ${ }^{1}$ In addition, the infection may cause prolonged or new-onset symptoms following infection. Although there is no universally accepted definition, this is often termed "long coronavirus disease" (long-COVID) and it may have a significant impact on social functioning, work capacity, and quality of life of patients. ${ }^{2,3}$

Early studies found that $10-13 \%$ of all SARS-CoV-2 positive individuals reported persistent symptoms for several months, ${ }^{2}$ which increased to $72-87 \%$ when examining only patients hospitalized with coronavirus disease 2019 (COVID-19). ${ }^{4-6}$ However, many of these reports are single-center studies or rely on voluntary reports of symptoms using, eg a smartphone application, which may introduce selection and information bias. ${ }^{4-7}$ In addition, the generalizability of hospitalized COVID-19 patients from countries with overwhelmed health-care systems during the very onset of the pandemic may be limited. Another study considered hospital diagnosis codes and medication prescriptions as proxies for long-COVID among SARS-CoV-2 positive compared with negative individuals and observed an increased risk of dyspnea and prescription of bronchodilating agents following infection. ${ }^{8}$ 
Health-care registries and databases are valuable tools for examining long-COVID from a population-based point of view. ${ }^{9-13}$ Yet, the validity of specific diagnosis codes for long-COVID remains unclear. This study aimed to examine the positive predictive value (PPV) of the International Classification of Diseases version 10 (ICD-10) code for long-COVID in the North Denmark Region.

\section{Methods}

\section{Setting}

Medical care is tax-supported and free of charge for all residents in Denmark. A unique civil registration number is assigned at birth, which allows for a unique identification of all Danish residents and an unambiguous linkage of individuals between registries. ${ }^{10}$ In the North Denmark Region, an outpatient clinic for patients with long-COVID was established on January 1, 2021 at Aalborg University Hospital (Figure 1). The catchment population was 590,439 (January 1, 2021) and 21,727 had tested positive for SARS-CoV-2 in the North Denmark Region during the study period. ${ }^{14,15}$ Patients eligible for consultation at the hospital outpatient clinic were required to have had symptoms for $>6$ weeks and a previous SARS-CoV-2 infection documented by a positive polymerase chain reaction (PCR) test on a respiratory sample or a positive serum antibody test. In addition, all patients admitted with acute COVID-19 infection requiring oxygen treatment were invited to a follow-up examination at Aalborg University Hospital.

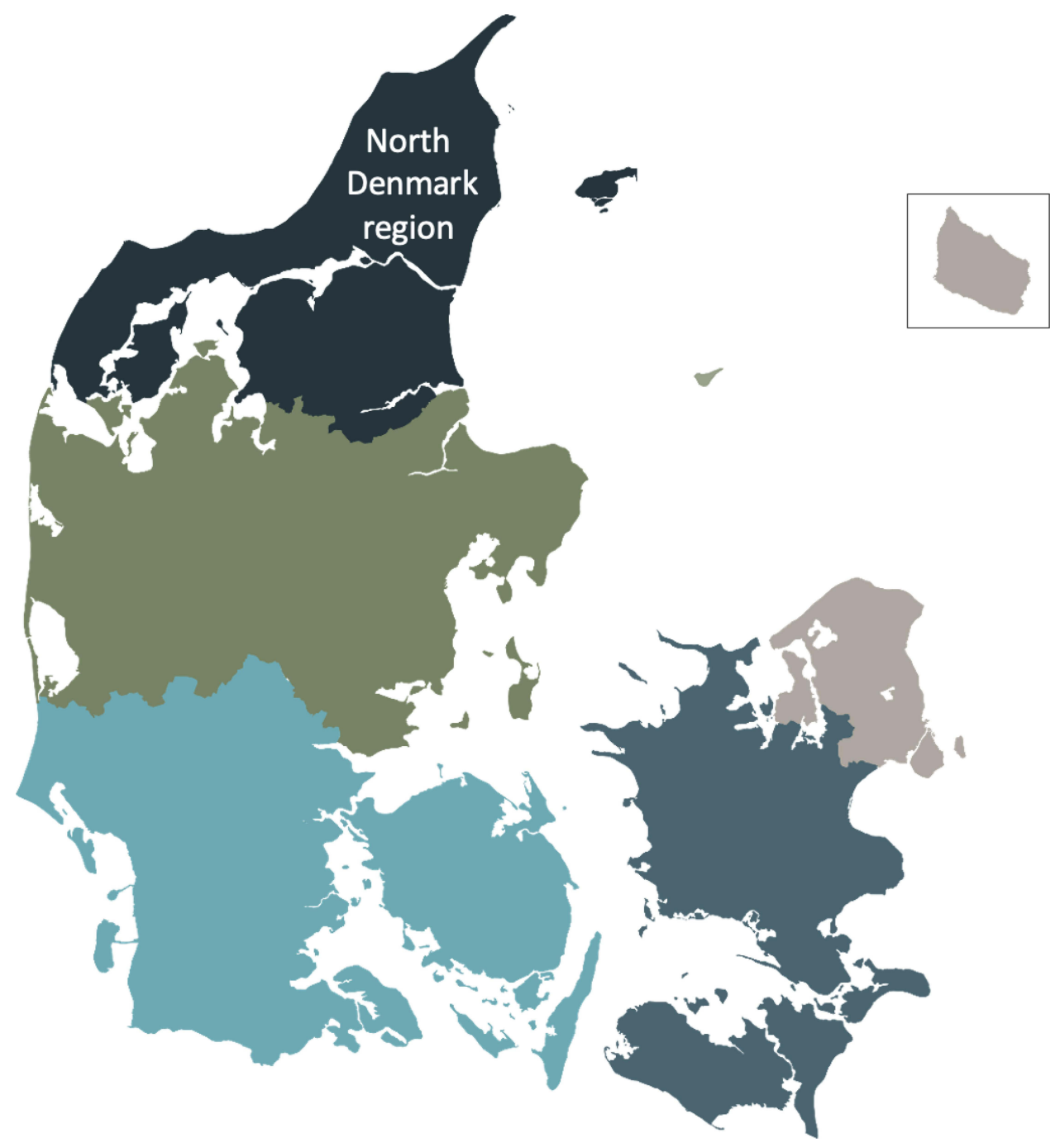

Figure I A map of the Denmark. The North Denmark Region is marked with black. 


\section{Study Design and Study Population}

This population-based cross-sectional validation study was conducted by reviewing the medical records of all patients assigned a first time hospital diagnosis code for long-COVID using the Danish version of ICD-10 (DB948A implemented April 1, 2020 by the Danish Board of Health) in North Denmark Region from February 27, 2020 through June 30, 2021 with censoring of follow-up by August 31, 2021. Data from primary care were not available for this study.

\section{Record Review and Definition of Long-COVID}

A local investigator (LSD) reviewed the medical records of all patients assigned a long-COVID diagnoses code including doctor's notes, laboratory results, and outcomes of patients. The date of positive polymerase chain reaction (PCR) test for SARS-CoV-2 was used as index date since the exact onset of long-COVID symptoms was often unclear and difficult to differentiate from acute COVID-19. However, for patients included only by a positive serum antibody test, time of longCOVID symptom onset as reported by the patients was used as index date.

Consistent with requirements for referral to the long-COVID outpatient clinic in the North Denmark Region during the study period, long-COVID was defined as the presence of all of the following criteria:

1. A positive PCR test for SARS-CoV-2 on a respiratory sample or a positive serum antibody test.

2. Symptoms suggestive of long-COVID (eg fatigue, neurocognitive deficits, headache, dyspnea, cough, gastrointestinal symptoms, chest pain, anosmia, or skin rashes) with no other diagnosis considered more likely based on all available information including patient history, physical and diagnostic examinations, laboratory results, imaging and, if needed, multidisciplinary consultations.

3. Symptom duration $>6$ weeks.

In cases of doubt, categorizations of patients were resolved by discussion with a senior specialist (JB). Patients were excluded if long-COVID had not been confirmed or disproven by August 31, 2021.

\section{Statistical Analysis}

Categorical variables were presented as $\mathrm{n} / \mathrm{N}(\%)$ and continuous variables as medians with interquartile ranges (IQR). Using the exact binomial method, the PPV with 95\% confidence intervals for long-COVID was calculated as the number of confirmed long-COVID cases divided by the number of patients assigned a diagnosis code of long-COVID. Next, PPVs were examined by sex, age groups $(0-17,18-40,41-60,61-80,81-)$, primary- and secondary diagnosis codes, and in-hospital treatment for acute COVID-19 infection (yes/no).

Study data were collected and managed using REDCap electronic data capture tools hosted at the North Denmark Region. ${ }^{16}$ Stata/MP ${ }^{\circledR}$ version 16 (StataCorp LLC, TX, USA) was used for all statistical analyses.

\section{Ethical Considerations}

This study was approved by the hospital administration as a quality of care project and patient consent or permission from an ethical committee was therefore not required for this type of study in Denmark. Handling of data complied with relevant data protection and privacy regulations and was conducted in accordance with the Declaration of Helsinki.

\section{Results}

A total of 306 patients were assigned a hospital diagnosis code for long-COVID corresponding to $1.4 \%$ of all SARS-CoV-2 positive patients in the North Denmark Region during the study period $(n=21,727)$ and yielding an incidence of 39/100000/ year. Of these, 40 patients were assigned a long-COVID diagnosis code before the establishment of the outpatient clinic at Aalborg University Hospital. Next, 40 patients were excluded due to incomplete diagnostic evaluation by August 31, 2021, leaving 266 patients for further analysis in this study (Figure 2). 


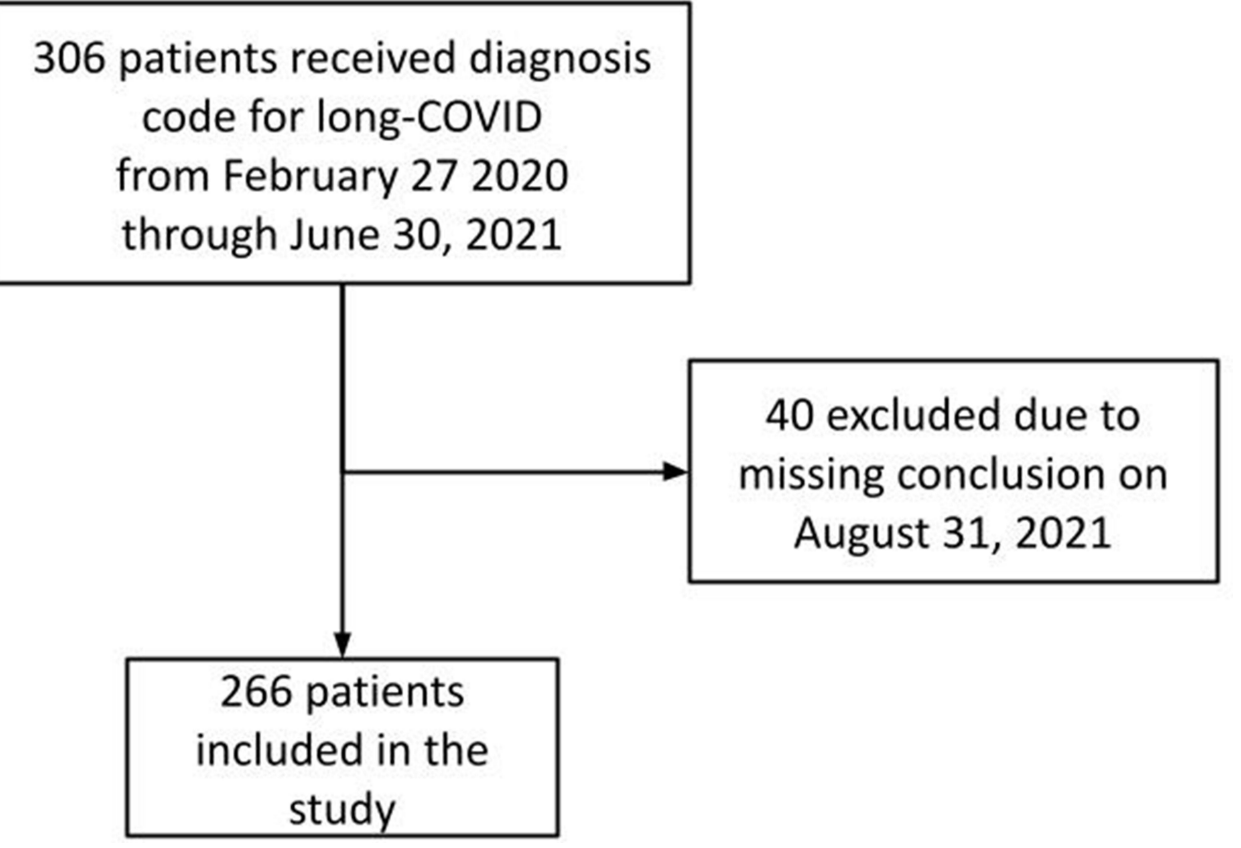

Figure 2 Flowchart of the study population.

Patients assigned a long-COVID hospital diagnosis code had a median age of 51 years (IQR: 43-60) and 34\% (91/ 266) were male. A total of 81/266 (30\%) had been hospitalized during acute COVID-19 infection with 32/266 (12\%) treated at the intensive care unit. The median duration of long-COVID symptoms was 148 days (IQR: 91-186) (Table 1).

The patients' symptoms were divided into constitutional and organ specific symptoms. One or more constitutional symptoms were present in 211/266 (79\%) patients, with fatigue 180/266 (68\%) as the most common. Other constitutional symptoms were attention/concentration difficulties 119/266 (45\%), headache 93/266 (35\%), insomnia 28/266 (11\%), dizziness 17/266 (6\%), and fever 6/266 (2\%). Organ-specific complaints consisted of pulmonary symptoms in 168/266 (63\%), earnose-and-throat (ENT) in 99/266 (37\%), musculoskeletal in 49/266 (18\%), cardiac in 34/266 (13\%), neurological in 16/266 (6\%), gastrointestinal in 18/266 (7\%), urogenital in 1/266 (0.4\%), and dermatological in 2/266 (0.8\%).

Overall, the diagnosis of long-COVID was confirmed in 249/266 yielding a PPV of 94\% (95\%CI: 90-96). The PPV was 96\% (95\%CI: 93-99) in females compared with 98\% in males (95\%CI: 79-94), and ranged from 98\% (95\%CI: 90-100) in those aged $18-40$ years to $86 \%$ (95\%CI: 73-94) in those aged 61-80 years. In the nine patients 81 years or older, the PPV was 44\% (95\%CI: 14-79) compared with 80\% (95\%CI: 28-99) in the five patients less than 18 years of age. The PPV was 96\% (95\%CI: 93-98) if long-COVID was a primary diagnosis code and 20\% (95\%CI: 2-56) for secondary diagnosis codes. Furthermore, the PPV was $84 \%$ (95\%CI: 74-91) for patients previously hospitalized for COVID-19 and 98\% (95\%CI: 9599) for those who had not been hospitalized for COVID-19 (Table 2). Using 12 weeks duration of symptoms as cut-off, longCOVID was confirmed in 201/205 patients corresponding to an overall PPV 98\% (95\%CI: 95-99).

Cases with disproven long-COVID consisted of patients hospitalized with COVID-19 (n=8) or re-admitted with dehydration $(n=3)$ or stroke $(n=2)$ shortly thereafter. The remaining cases were diagnosed with polymyalgia rheumatica $(\mathrm{n}=1)$, asthma $(\mathrm{n}=1)$, underlying cardiac disease $(\mathrm{n}=1)$, and deep venous thrombosis $(\mathrm{n}=1)$ instead of long-COVID.

\section{Discussion}

In this study, a high PPV of the long-COVID diagnosis code was observed overall and across most subgroups including sex, age, and previous hospitalization for acute COVID-19 infection. However, caution is advised when using longCOVID diagnosis codes as secondary codes and in children and adolescents as well as the very elderly due to small sample sizes in these groups. 
Table I Baseline Characteristics of 266 Patients Assigned a First-time ICD-10 Diagnosis Code for Long-COVID, in the North Denmark Region from February 27, 2020 Through June 30, 2021

\begin{tabular}{|c|c|}
\hline Characteristic & n (\%) or Median (IQR) \\
\hline \multicolumn{2}{|l|}{ Sex } \\
\hline Females & $175(66)$ \\
\hline Males & $91(34)$ \\
\hline \multicolumn{2}{|l|}{ Department of long-COVID diagnosis } \\
\hline Long-COVID clinic (Aalborg University Hospital) & $232(87)$ \\
\hline Internal medicine department & $27(10)$ \\
\hline Pediatric department & $5(2)$ \\
\hline Neurological department & $2(1)$ \\
\hline Age, years & $51(43-60)$ \\
\hline \multicolumn{2}{|l|}{ Age group, years } \\
\hline $0-17$ & $5(2)$ \\
\hline $18-40$ & $51(19)$ \\
\hline $4 I-60$ & $152(57)$ \\
\hline $6 I-80$ & $49(19)$ \\
\hline $81-$ & $9(3)$ \\
\hline \multicolumn{2}{|l|}{ Admission to hospital during acute COVID-19 } \\
\hline Yes & $81(30)$ \\
\hline No & $185(70)$ \\
\hline \multicolumn{2}{|l|}{ Admission to ICU during acute COVID-I9 } \\
\hline Yes & $32(12)$ \\
\hline No & $234(88)$ \\
\hline Duration of symptoms, days & $148(9|-| 86)$ \\
\hline \multicolumn{2}{|l|}{ Symptoms } \\
\hline Constitutional & $211(79)$ \\
\hline Headache & $93(35)$ \\
\hline Fatigue & $180(68)$ \\
\hline Attention/concentration difficulties & $119(45)$ \\
\hline Fever & $6(2)$ \\
\hline Dizziness & $17(6)$ \\
\hline Insomnia & $28(\mathrm{II})$ \\
\hline \multicolumn{2}{|l|}{ Organ specific symptoms } \\
\hline Pulmonary & $168(63)$ \\
\hline ENT & $99(37)$ \\
\hline Musculoskeletal & $49(18)$ \\
\hline Cardiac & $34(13)$ \\
\hline Neurological & $16(8)$ \\
\hline Gastrointestinal & $18(7)$ \\
\hline Urogenital & $\mathrm{I}(0.4)$ \\
\hline Skin & $2(0.8)$ \\
\hline
\end{tabular}

Abbreviations: COVID-19, coronavirus disease 2019; ENT, ear-nose-throat.

Long-COVID is still a very novel disease and the scientific community, health-care administrators, and public opinion leaders have voiced concern about the potential impact as SARS-CoV-2 continues to spread globally and new variants evolve that may evade previous natural or vaccine-induced immunity. ${ }^{17-19}$ Predictors of long-COVID have been suggested to include female sex, increasing number of symptoms during COVID-19, number of preexisting comorbidities, median SARS-CoV-2 IgG titers, and intensive care unit admission. ${ }^{19-21}$ Treatment has thus far mainly focused on physiotherapy and occupational rehabilitation for constitutional symptoms. ${ }^{2}$ 
Table 2 Positive Predictive Value (PPV) with 95\% Confidence Intervals (CI) of ICD-I0 Diagnosis Code for Long-COVID in the North Denmark Region

\begin{tabular}{|l|c|c|}
\hline Long COVID & Number of Observations (n/N) & PPV \% (95\%CI) \\
\hline Confirmed overall & $249 / 266$ & $94(90-96)$ \\
\hline Sex & & \\
\hline Females & $169 / 175$ & $97(93-99)$ \\
\hline Males & $80 / 91$ & $88(79-94)$ \\
\hline Age group & & \\
\hline $0-17$ & $4 / 5$ & $80(28-99)$ \\
\hline I8-40 & $50 / 51$ & $98(90-100)$ \\
\hline $4 I-60$ & $149 / 152$ & $98(94-100)$ \\
\hline $6 I-80$ & $42 / 49$ & $86(73-94)$ \\
\hline $8 I-$ & $4 / 9$ & $44(14-79)$ \\
\hline Primary diagnosis code & $247 / 256$ & $96(93-98)$ \\
\hline Secondary diagnosis code & $2 / 10$ & $20(2-56)$ \\
\hline Previous in-hospital admission for COVID-19 & & $98(95-99)$ \\
\hline Yes & $181 / 185$ & \\
\hline No & & \\
\hline
\end{tabular}

Health-care registries may prove helpful by providing population-based estimates on incidence of long-COVID and associated morbidity requiring treatment at hospitals as well as socioeconomic consequences of long-COVID. ${ }^{9-13}$ However, a key prerequisite is that the information registered in these databases is accurate and, to the best of our knowledge, the ICD-10 diagnosis code for long-COVID has not yet been validated in other settings. Of note, the demographic composition and clinical presentation of long-COVID patients in the current study was comparable to other reports on long-COVID. ${ }^{20-22}$ Several previous studies on long-COVID were conducted before the availability of specific diagnosis codes for long-COVID or before outpatient clinics were established to manage these complex patients. ${ }^{8,23}$ Moreover, most studies have been based on clinical cohorts with inherent limitations in sample size and followup. ${ }^{4-6,21,22}$ These combined considerations highlight the need for additional registry-based research using specific and validated diagnosis codes in large population-based cohorts of previously hospitalized and nonhospitalized COVID-19 patients with matched control groups in order to learn more about long-COVID and the potential consequences of the disease.

This study has limitations. Importantly, an internationally accepted scientific and clinical definition for long-COVID has still not been developed and validated. As an example, the requirement for duration of symptoms varies from just four weeks to 12 weeks. ${ }^{2,3,24}$ If future definitions should deviate substantially from the one used in this study, the results may not be applicable. ${ }^{2,3}$ In contrast, the predefined criteria in this study limited ascertainment bias when assessing the medical records and enables comparison with other settings. Since 40 patients with a long-COVID diagnosis code were excluded due to incomplete diagnostic evaluation, caution is advised for allowing adequate follow-up time after a longCOVID diagnosis code to ensure correct categorization of patients depending on results of diagnostic workup. Additional information bias may also be present since the diagnostic workup was left at the discretion of the physician in charge. However, local guidelines for management of long-COVID patients were implemented by February 2021 in the North 
Denmark Region, which support a uniform diagnostic workup of patients. Although the population-based setting of the North Denmark Region ensured complete evaluation of all patients with a hospital diagnosis code for long-COVID, selection bias cannot be ruled out since only patients with a proven SARS-CoV-2 infection were accepted to the outpatient clinic. The study was not able to assess the sensitivity of the long-COVID diagnosis code as it would require medical record review of all hospitalized patients in the North Denmark Region during the study period, which was not feasible. Finally, coding practices may vary between regions in Denmark, but the generalizability is still considered to be high due to the population-based study design and the tax-financed health-care system providing free medical care at the point of delivery for all Danish residents. ${ }^{11}$

In conclusion, the overall PPV of the ICD-10 diagnosis code for long-COVID was high in our cohort and suitable for future registry-based studies.

\section{Abbreviations}

CI, confidence interval; COVID-19, coronavirus disease 2019; ICD-10, International Classification of Diseases version 10; PPV, positive predictive value; SARS-CoV-2, severe acute respiratory syndrome coronavirus 2.

\section{Funding}

There is no funding to report.

\section{Disclosure}

The authors report no conflicts of interest in this work.

\section{References}

1. Ritchie H, Mathieu E, Rodés-Guirao L, et al. Coronavirus (COVID-19) cases. OurWorldInData.org. Available from: https://ourworldindata.org/ coronavirus. Accessed December 10, 2021.

2. Shah W, Hillman T, Playford ED, Hishmeh L. Managing the long term effects of covid-19: summary of NICE, SIGN, and RCGP rapid guideline. BMJ. 2021;372. doi:10.1136/bmj.n136

3. Greenhalgh T, Knight M, A'Court C, Buxton M, Husain L. Management of post-acute covid-19 in primary care. BMJ. 2020;370. doi:10.1136/bmj. $\mathrm{m} 3026$

4. Mandal S, Barnett J, Brill SE, et al. Long-COVID': a cross-sectional study of persisting symptoms, biomarker and imaging abnormalities following hospitalisation for COVID-19. Thorax. 2021;76(4):396-398. doi:10.1136/thoraxjnl-2020-215818

5. Carfi A, Bernabei R, Landi F. Persistent symptoms in patients after acute COVID-19. JAMA. 2020;324(6):603-605. doi:10.1001/jama.2020.12603

6. Huang C, Huang L, Wang Y, et al. 6-month consequences of COVID-19 in patients discharged from hospital: a cohort study. Lancet. 2021;397 (10270):220-232. doi:10.1016/S0140-6736(20)32656-8

7. Sudre CH, Murray B, Varsavsky T, et al. Attributes and predictors of long COVID. Nat Med. 2021:626-631. doi:10.1038/s41591-021-01292-y

8. Lund LC, Hallas J, Nielsen H, et al. Post-acute effects of SARS-CoV-2 infection in individuals not requiring hospital admission: a Danish population-based cohort study. Lancet Infect Dis. 2021;21(10):1373-1382. doi:10.1016/S1473-3099(21)00211-5

9. Johannesdottir SA, Horváth-Puhó E, Ehrenstein V, Schmidt M, Pedersen L, Sørensen HT. Existing data sources for clinical epidemiology: the Danish National database of reimbursed prescriptions. Clin Epidemiol. 2012;4(1):303-313. doi:10.2147/clep.s37587

10. Schmidt M, Schmidt SAJ, Sandegaard JL, Ehrenstein V, Pedersen L, Sørensen HT. The Danish National patient registry: a review of content, data quality, and research potential. Clin Epidemiol. 2015;7:449-490. doi:10.2147/CLEP.S91125

11. Schmidt M, Pedersen L, Sørensen HT. The Danish Civil Registration System as a tool in epidemiology. Eur J Epidemiol. 2014;29(8):541-549. doi:10.1007/s10654-014-9930-3

12. Sørensen HT, Pedersen L, Jørgensen J, Ehrenstein V. Danish clinical quality databases - an important and untapped resource for clinical research. Clin Epidemiol. 2016;8:425-427. doi:10.2147/CLEP.S113265

13. Schmidt M, Schmidt SAJ, Adelborg K, et al. The Danish health care system and epidemiological research: from health care contacts to database records. Clin Epidemiol. 2019;11:563-591. doi:10.2147/CLEP.S179083

14. Danmarks Statistik (Statistics Denmark). Population census. Available from: https://statbank.dk/statbank5a/default.asp?w=1680. Accessed October 14, 2021.

15. Statens Serum Institut. Covid-19 data. Available from: https://experience.arcgis.com/experience/aa41b29149f24e20a4007a0c4e13db1d. Accessed October 14, 2021.

16. Harris PA, Taylor R, Thielke R, Payne J, Gonzalez N, Conde JG. Research electronic data capture (REDCap)-A metadata-driven methodology and workflow process for providing translational research informatics support. J Biomed Inform. 2009;42(2):377-381. doi:10.1016/j.jbi.2008.08.010

17. Yelin D, Wirtheim E, Vetter P, et al. Long-term consequences of COVID-19: research needs. Lancet Infect Dis. 2020;20(10):1115-1117. doi:10.1016/S1473-3099(20)30701-5

18. Norton A, Olliaro P, Sigfrid L, et al. Long COVID: tackling a multifaceted condition requires a multidisciplinary approach. Lancet Infect Dis. 2021;21(5):601-602. doi:10.1016/S1473-3099(21)00043-8 
19. Jiang DH, Roy DJ, Gu BJ, Hassett LC, McCoy RG. Postacute sequelae of severe acute respiratory syndrome Coronavirus 2 infection. JACC. 2021. doi:10.1016/j.jacbts.2021.07.002

20. Peghin M, Palese A, Venturini M, et al. Post-COVID-19 symptoms 6 months after acute infection among hospitalized and non-hospitalized patients. Clin Microbiol Infect. 2021;27(10):1507-1513. doi:10.1016/j.cmi.2021.05.033

21. Stavem K, Ghanima W, Olsen MK, Gilboe HM, Einvik G. Persistent symptoms 1.5-6 months after COVID-19 in non-hospitalised subjects: a population-based cohort study. Thorax. 2021;76(4):405-407. doi:10.1136/thoraxjnl-2020-216377

22. Zayet S, Zahra H, Royer PY, et al. Post-COVID-19 syndrome: nine months after SARS-CoV-2 infection in a cohort of 354 patients: data from the first wave of COVID-19 in nord franche-comté hospital, France. Microorganisms. 2021;9(8):1719. doi:10.3390/microorganisms9081719

23. Leth S, Gunst JD, Mathiasen V, et al. Persistent symptoms in patients recovering from COVID-19 in Denmark. Open Forum Infect Dis. 2021;8(4). doi:10.1093/ofid/ofab042

24. Centers for Disease Control and Prevention. Post-COVID conditions. Available from: https://www.cdc.gov/coronavirus/2019-ncov/long-termeffects/index.html. Accessed December 10, 2021.

\section{Publish your work in this journal}

Clinical Epidemiology is an international, peer-reviewed, open access, online journal focusing on disease and drug epidemiology, identification of risk factors and screening procedures to develop optimal preventative initiatives and programs. Specific topics include: diagnosis, prognosis, treatment, screening, prevention, risk factor modification, systematic reviews, risk \& safety of medical interventions, epidemiology \& biostatistical methods, and evaluation of guidelines, translational medicine, health policies \& economic evaluations. The manuscript management system is completely online and includes a very quick and fair peer-review system, which is all easy to use. 